\title{
Estimate Stress-Strength Reliability Model Using Rayleigh and Half-Normal Distribution
}

\author{
Osama Abdulaziz Alamri, ${ }^{1}$ M. M. Abd El-Raouf $\mathbb{D}^{2},{ }^{2}$ Eman Ahmed Ismail, ${ }^{3}$ \\ Zahra Almaspoor $\mathbb{D}^{4}{ }^{4}$ Basim S. O. Alsaedi $\mathbb{D}^{1},{ }^{1}$ Saima Khan Khosa, ${ }^{5}$ and M. Yusuf $\mathbb{D}^{6}$ \\ ${ }^{1}$ Department of Statistics, Faculty of Science, University of Tabuk, Tabuk 71491, Saudi Arabia \\ ${ }^{2}$ Basic and Applied Science Institute, Arab Academy for Science, Technology and Maritime Transport (AASTMT), \\ Alexandia, Egypt \\ ${ }^{3}$ College of International Transport and Logistics, \\ Arab Academy for Science, Technology and Maritime Transport, Smart Village Campus, Cairo, Egypt \\ ${ }^{4}$ Department of Statistics, Yazd University, P.O. Box 89175-741, Yazd, Iran \\ ${ }^{5}$ Department of Statistics, Bahauddin Zakariya University, Multan, Pakistan \\ ${ }^{6}$ Department of Mathematics, Faculty of science, Helwan University, Cairo, Egypt
}

Correspondence should be addressed to Zahra Almaspoor; z.almaspoor@stu.yazd.ac.ir

Received 25 May 2021; Accepted 23 June 2021; Published 5 July 2021

Academic Editor: Ahmed Mostafa Khalil

Copyright (C) 2021 Osama Abdulaziz Alamri et al. This is an open access article distributed under the Creative Commons Attribution License, which permits unrestricted use, distribution, and reproduction in any medium, provided the original work is properly cited.

\begin{abstract}
In the field of life testing, it is very important to study the reliability of any component under testing. One of the most important subjects is the "stress-strength reliability" term which always refers to the quantity $P(X>Y)$ in any statistical literature. It resamples a system with random strength $(X)$ that is subjected to a random strength $(Y)$ such that a system fails in case the stress exceeds the strength. In this study, we consider stress-strength reliability where the strength $(X)$ follows Rayleigh-half-normal distribution and stress $\left(Y_{1}, Y_{2}, Y_{3}\right.$, and $\left.Y_{4}\right)$ follows Rayleigh-half-normal distribution, exponential distribution, Rayleigh distribution, and half-normal distribution, respectively. This effort comprises determining the general formulations of the reliabilities of a system. Also, the maximum likelihood estimation approach and method of moment (MOM) will be utilized to estimate the parameters. Finally, reliability has been attained utilizing various values of stress and strength parameters.
\end{abstract}

\section{Introduction}

The life of a component is described using the stress-strength models, in reliability theory, that is including a random strength $(X)$ which is subjected to a random stress $(Y)$. The failure of a component is occurred instantaneously when the stress level applied to it exceeds the level of the strength. Thus, the component reliability is measured by $R=P(Y<X)$. This measurement has a variety of applications, most notably in the engineering industry, such as the degradation of rocket motors and structures, the fatigue failure of aircraft structures, the ageing of concrete pressure vessels, and static fatigue of ceramic components. Therefore, the estimation of $R=P(Y<X)$ has a great importance in the practical applications. The literature demonstrates that reliability estimation $(R)$ has already been performed when the distributions of $(X)$ and $(Y)$ are Weibull, exponential, or log normal.

Church and Harris [1] firstly introduced the term stressstrength. Many authors have adopted various distributions types for stress and strength. The works of Church and Harris, Surles and Padgett [2], Raqab and Kundu [3], Mokhlis [4], and Saraçoğlu et al. [5] contain the discussion of the estimation problems of the stress-strength reliability model for different distributions. Recently, a review of all methods and results on the stress-strength reliability have presented by Kotz et al. [6]. Bayes estimators and reliability function and the parameters of the Consul, Geeta, and size- 
biased Geeta distributions are obtained by Khan Adil and Jan [7]. Akman et al. [8] studied the estimation of reliability using a finite mixture of inverse Gaussian distributions. The estimation of $R=P(Y<X)$ is studied by AI-Hussaini [9] based on a finite mixture of lognormal components. For more reading, see [10-14].

\section{Finite Mixture of Rayleigh and Half- Normal Distribution}

The Rayleigh-half-normal distribution is denoted as $\mathbf{R H N}(\theta)$ by Abd El-Monsef and Abd El-Raouf [15]. A mixture of Rayleigh and half-normal distribution with a parameter $(1 / \sqrt{2 \theta})$ is used to represent this model:

$$
\begin{aligned}
f(x, \theta) & =K f_{R}\left(\frac{x \cdot 1}{\sqrt{2 \theta}}\right)+(1-K) f_{\mathrm{HN}}\left(\frac{x \cdot 1}{\sqrt{2 \theta}}\right) \\
& =K\left(2 \theta x e^{-\theta x^{2}}\right)+(1-K)\left(2 \sqrt{\frac{\theta}{\pi}} e^{-\theta x^{2}}\right),
\end{aligned}
$$

where $K=(1 /(1+\sqrt{\pi \theta}))$.

Thus, the Rayleigh-half normal distribution probability density function (pdf) is given by

$$
f(x, \theta)=\frac{2 \theta(x+1) e^{-\theta x^{2}}}{1+\sqrt{\pi \theta}}, \quad x, \theta>0 .
$$

The corresponding cumulative distribution function is given by

$$
F(x, \theta)=\frac{1-e^{-\theta x^{2}}+\sqrt{\pi \theta} \operatorname{erf}(\sqrt{\theta} x)}{1+\sqrt{\pi \theta}}, \quad x, \theta>0,
$$

where $\operatorname{erf}(u)$ is the Gauss error function defined as

$$
\operatorname{erf}(u)=\frac{2}{\sqrt{\pi}} \int_{0}^{u} e^{-t^{2}} \mathrm{~d} t
$$

2.1. The Survival Function and the Hazard Function. The reliability function or the survival function $S(x)$ tests the chance of occurring of a breakdown of units beyond certain given point in time. For monitoring, a unit lifetime across the support of its lifetime distribution; generally, the probability that an item will work properly for a specified time period with no failure is the survival function. The definition of the survival function is represented as follows:

$$
S(x)=1-F(x)=\frac{e^{-\theta x^{2}}+\sqrt{\pi \theta} \operatorname{erfc}(\sqrt{\theta} x)}{1+\sqrt{\pi \theta}},
$$

where $\operatorname{erfc}(u)$ is the complementary error function, and its definition is

$$
\operatorname{erfc}(u)=1-\operatorname{erf}(u)=\frac{2}{\sqrt{\pi}} \int_{u}^{\infty} e^{-t^{2}} \mathrm{~d} t
$$

The definition of the hazard rate function is the ratio between the density function and its survival function, which measures the tendency to die or to fail depending on the reached age, and therefore, it has a critical role in the classification of the distributions of lifetime, so the hazard rate function of the RHN distribution is given by

$$
h(x)=\frac{f(x)}{S(x)}=\frac{2 \theta(1+x)}{1+e^{\theta x^{2}} \sqrt{\pi \theta} \operatorname{erf}(\sqrt{\theta} x)} .
$$

\section{Stress-Strength Reliability Computations}

In this section, the reliability $R=P(Y<X)$ was derived, where the random variables $(X)$ and $(Y)$ are the independent random variables, where the strength $X$ follows Rayleighhalf normal distribution and the stress $(Y)$ takes different cases (Rayleigh-half normal distribution, exponential distribution, Rayleigh distribution, and half-normal distribution).

Let $(X)$ and $(Y)$ be two independent random variables, where $(X)$ represents "strength" and $(Y)$ represents "stress" and $(X)$, and $(Y)$ follows a joint pdf $f(x, \theta)$; thus, the component reliability is

$$
R=P(Y<X)=\int_{-\infty}^{\infty} \int_{-\infty}^{x} f(x, y) \mathrm{d} y \mathrm{~d} x
$$

In case that the random variables are statistically independent, then $f(x, y)=f(x) g(y)$ so that

$$
R=\int_{-\infty}^{\infty} \int_{-\infty}^{x} f(x) g(x) \mathrm{d} y \mathrm{~d} x
$$

where $f(x)$ and $g(y)$ are pdfs of $X$ and $Y$, respectively.

3.1. The Stress and the Strength Follows Rayleigh-Half-Normal Distribution. As the strength $X \sim \operatorname{RHN}(\theta)$ and $Y_{1} \sim \operatorname{RHN}\left(\theta_{1}\right)$, they are independent random variables with pdf $f(x)$ and $g\left(y_{1}\right)$, respectively:

$$
\begin{gathered}
f(x)=\frac{2 \theta(x+1) e^{-\theta x^{2}}}{1+\sqrt{\pi \theta}}, \quad 0<\theta \cdot x \\
g\left(y_{1}\right)=\frac{2 \theta_{1}\left(y_{1}+1\right) e^{-\theta_{1} y_{1}^{2}}}{1+\sqrt{\pi \theta_{1}}}, \quad 0<\theta_{1} \cdot y_{1} .
\end{gathered}
$$

We derive the reliability $R=P(Y<X)$ as follows: 


$$
\begin{aligned}
R_{1} & =P(Y<X)=\int_{0}^{\infty} \int_{0}^{x} f(x) g\left(y_{1}\right) \mathrm{d} y \mathrm{~d} x \\
& =\int_{0}^{\infty} \int_{0}^{x}\left(\frac{2 \theta_{1}\left(y_{1}+1\right) e^{-\theta_{1} y_{1}^{2}}}{1+\sqrt{\pi \theta_{1}}}\right)\left(\frac{2 \theta(x+1) e^{-\theta x^{2}}}{1+\sqrt{\pi \theta}}\right) \mathrm{d} y \mathrm{~d} x .
\end{aligned}
$$

And, we get after the simplification:

$$
R_{1}=\frac{\left(1+\sqrt{\pi \theta_{1}}+2 \sqrt{\theta \theta_{1}} \operatorname{Tan}^{-1}\left(\sqrt{\theta / \theta_{1}}\right)+\left(\sqrt{\pi}\left(\theta-\theta_{1}\right) / \sqrt{\theta+\theta_{1}}\right)-\left(\theta_{1} /\left(\theta+\theta_{1}\right)\right)\right)}{1+\sqrt{\pi \theta}+\sqrt{\pi \theta_{1}}+\pi \sqrt{\theta \theta_{1}}} .
$$

3.2. The Strength Follows RHN Distribution and the Stress Follows Exponential Distribution. In this case, the probability density function (pdf) for the stress $Y_{2}$ that follows the exponential distribution is given by

$$
g\left(y_{2}\right)=\theta_{2} e^{-y_{1} \theta_{2}}, \quad y_{2}, \theta_{2}>0
$$

Then, reliability function $R_{2}$ for the independent random variables $X$ and $Y_{2}$ :

$$
\begin{aligned}
& R_{2}=\int_{0}^{\infty} \int_{0}^{x} \theta_{2} e^{-y_{2} \theta_{2}}\left(\frac{2 \theta(x+1) e^{-\theta x^{2}}}{1+\sqrt{\pi \theta}}\right) \mathrm{d} y \mathrm{~d} x, \\
& R_{2}=\frac{\sqrt{\pi}}{2(\sqrt{\theta}+\sqrt{\pi} \theta)}\left(2 \theta-\left(2 \theta-\theta_{2}\right) e^{\left(\theta_{2}^{2} / 4 \theta\right)} \operatorname{erfc}\left(\frac{\theta_{2}}{2 \sqrt{\theta}}\right)\right),
\end{aligned}
$$

where the strength follows RHN distribution.

3.3. The Strength Follows RHN Distribution and the Stress Follows Rayleigh Distribution. In this case, the probability density function (pdf) for the stress $Y_{3}$ that follows the Rayleigh distribution is given by

$$
g\left(y_{3}\right)=\frac{y_{3}}{\theta_{2}^{2}} e^{-\left(y_{3}^{2} / 2 \theta_{3}^{2}\right)}, \quad y, \theta_{3}>0
$$

Then, reliability function $R_{3}$ for the independent random variables $X$ and $Y_{3}$ is

$$
\begin{aligned}
R_{3} & =\int_{0}^{\infty} \int_{0}^{x}\left(\frac{y_{3}}{\theta_{3}^{2}} e^{-\left(y_{3}^{2} / 2 \theta_{3}^{2}\right)}\right)\left(\frac{2 \theta(x+1) e^{-\theta x^{2}}}{1+\sqrt{\pi \theta}}\right) \mathrm{d} y \mathrm{~d} x \\
& =\frac{2 \theta}{(1+\sqrt{\pi \theta})} \int_{0}^{\infty}(x+1) e^{-\theta x^{2}}\left(1-e^{-\left(x^{2} / 2 \theta_{3}^{2}\right)}\right) \mathrm{d} x \\
R_{3} & =\frac{\theta}{1+\sqrt{\pi \theta}}\left(\frac{1}{\theta}+\frac{\sqrt{\pi}}{\sqrt{\theta}}-\frac{2}{2 \theta+\left(1 / \theta_{3}^{2}\right)}-\frac{\sqrt{2 \pi}}{\sqrt{2 \theta+\left(1 / \theta_{3}^{2}\right)}}\right),
\end{aligned}
$$

where the strength follows RHN distribution.

3.4. The Strength Follows RHN Distribution and the Stress Follows Half-Normal Distribution. In this case, the probability density function (pdf) for the stress $Y_{4}$ that follows half-normal distribution is given by

$$
g\left(y_{4}\right)=\left(\frac{\sqrt{2}}{\theta_{4} \sqrt{\pi}}\right) e^{-\left(y_{4}^{2} / 2 \theta_{4}^{2}\right)}, \quad y, \theta_{4}>0 .
$$

Then, reliability function $R_{4}$ for the independent random variables $X$ and $Y_{4}$

$$
\begin{aligned}
R_{4} & =\int_{0}^{\infty} \int_{0}^{x}\left(\left(\frac{\sqrt{2}}{\theta_{4} \sqrt{\pi}}\right) e^{-\frac{y_{4}^{2}}{2 \theta_{4}^{2}}}\right)\left(\frac{2 \theta(x+1) e^{-\theta x^{2}}}{1+\sqrt{\pi \theta}}\right) \mathrm{d} y \mathrm{~d} x \\
& =\frac{2 \theta}{(1+\sqrt{\pi \theta})} \int_{0}^{\infty} \operatorname{Erf}\left(\frac{x}{\sqrt{2} \theta_{4}}\right)(x+1) e^{-\theta x^{2}} \mathrm{~d} x, \\
R_{4} & =\frac{1}{\sqrt{\theta}(1+\sqrt{\pi \theta})}\left(\frac{2 \theta \operatorname{Cot}^{-1}\left(\theta_{4} \sqrt{2 \theta}\right)}{\sqrt{\pi}}+\frac{1}{\theta_{4} \sqrt{2+\left(1 / \theta \theta_{4}^{2}\right)}}\right),
\end{aligned}
$$

where the strength follows RHN distribution. 


\section{Estimation of Stress-Strength Reliability}

In the literature, a discussion of the estimation $R=P(Y<X)$ when random variables $(X)$ and $(Y)$ are following the specified distributions have been presented including engineering statistics, quality control, medicine, reliability, biostatistics, and psychology. This quantity for a limited number of cases could be calculated in a closed form (Nadarajah [16] and Barreto-Souza et al. [17]). Several authors including Milan and Vesna [18] have considered the estimation of $(R)$ for independent variables and normally distributed $(X)$ and $(Y)$. Later, a list of papers related to the estimation problem of $(R)$ were reported by Greco and Venture [19] when $(X)$ and $(Y)$ are independent and follow a class of lifetime distributions containing Gamma distributions, exponential, generalized exponential, bivariate exponential, Weibull distribution, Burr type $t$ model, and others.

4.1. Method of Moment (MOM) Estimation of $R$. The estimation of reliability is very common in the statistical literature. Now, to compute $\widehat{R}$, we need to estimate the parameters $\theta$ and $\theta_{i}, i=1,2,3,4$, in four cases of stress.

Since the strengths $X$ follow $\operatorname{RHN}(\theta)$, the stress have four cases:

(i) $Y_{1}$ follows Rayleigh-half normal distribution with parameter $\theta_{1}$ (ii) $Y_{2}$ follows exponential distribution with parameter $\theta_{2}$

(iii) $Y_{3}$ follows Rayleigh distribution with parameter $\theta_{3}$

(iv) $Y_{4}$ follows half-normal distribution with parameter $\theta_{4}$; then, their population means are given by

$$
\begin{aligned}
& \bar{x}=\frac{2 \sqrt{\theta}+\sqrt{\pi}}{2 \sqrt{\theta}(1+\sqrt{\theta \pi})}, \\
& \bar{y}_{1}=\frac{2 \sqrt{\theta_{1}}+\sqrt{\pi}}{2 \sqrt{\theta_{1}}\left(1+\sqrt{\theta_{1} \pi}\right)}, \\
& \bar{y}_{2}=\frac{1}{\theta_{2}}, \\
& \bar{y}_{3}=\theta_{3} \sqrt{\frac{\pi}{2}} \\
& \bar{y}_{4}=\theta_{4} \sqrt{\frac{2}{\pi}} .
\end{aligned}
$$

The ME's of $\theta, \theta_{1}, \theta_{2}, \theta_{3}$, and $\theta_{4}$, denoted by $\hat{\theta}, \widehat{\theta}_{1}, \widehat{\theta}_{2}, \widehat{\theta}_{3}$, and $\widehat{\theta}_{4}$, respectively, can be obtained by solving $\left(\bar{x}, \bar{y}_{1}, \bar{y}_{2}, \bar{y}_{3}\right.$, and $\left.\bar{y}_{4}\right)$ numerically:

$$
\begin{aligned}
\widehat{\theta} & =\frac{\left(\sum_{i=1}^{m} x_{i}-n\right)^{2}+n \pi \sum_{i=1}^{m} x_{i}}{2 \pi\left(\sum_{i=1}^{m} x_{i}\right)^{2}}+\frac{\sqrt{\left(\sum_{i=1}^{m} x_{i}-n\right)^{4}+2 n \pi \sum_{i=1}^{m} x_{i}\left(\sum_{i=1}^{m} x_{i}-n\right)^{2}}}{2 \pi\left(\sum_{i=1}^{m} x_{i}\right)^{2}}, \\
\widehat{\theta}_{1} & =\frac{\left(\sum_{j=1}^{m} y_{1_{j}}-m\right)^{2}+m \pi \sum_{j=1}^{m} y_{1_{j}}}{2 \pi\left(\sum_{j=1}^{m} y_{1_{j}}\right)^{2}}+\frac{\sqrt{\left(\sum_{j=1}^{m} y_{1_{j}}-n\right)^{4}+2 m \pi \sum_{j=1}^{m} y_{1_{j}}\left(\sum_{j=1}^{m} y_{1_{j}}-m\right)^{2}}}{2 \pi\left(\sum_{j=1}^{m} y_{1_{j}}\right)^{2}}, \\
\widehat{\theta}_{2} & =\frac{m}{\sum_{j=1}^{m} y_{2 j}}, \\
\widehat{\theta}_{3} & =\sqrt{\frac{2}{\pi}\left(\frac{\sum_{j=1}^{m} y_{3 j}}{m}\right),} \\
\widehat{\theta}_{4} & =\sqrt{\frac{\pi}{2}}\left(\frac{\sum_{j=1}^{m} y_{4 j}}{m}\right) .
\end{aligned}
$$

The ME of $R$, denoted by $\widehat{R}_{1} \cdot \widehat{R}_{2} \cdot \widehat{R}_{3}$ and $\widehat{R}_{4}$ is obtained by substitute $\hat{\theta}$ with $\widehat{\theta}_{1} \cdot \widehat{\theta}_{2} \cdot \widehat{\theta}_{3}$ and $\hat{\theta}_{4}$ in $R_{1} \cdot R_{2} \cdot R_{3}$ and $R_{4}$.

4.2. The Maximum Likelihood Estimators of $R$. The maximum likelihood estimator (MLE) is the most popular method for reliability estimation $R=p(Y<X)$ because of its generality and flexibility. This method can be used if the joint distribution of the strength $(X)$ and the stress $(Y)$ is a known function with some unknown parameters.

Suppose $x_{1} \cdot x_{2} \cdots x_{n}$ is a random sample from RHN distribution with $\theta$ and $y_{11} \cdot y_{12} \cdots y_{1 m}$ is a random sample from RHN distribution with $\theta_{1}$. Then, the likelihood function is given by 


$$
L\left(\theta \cdot \theta_{1} ; x \cdot y_{1}\right)=2^{n+m} \theta^{n} \theta_{1}^{m}-(1+\sqrt{\pi \theta})^{n}-\left(1+\sqrt{\pi \theta_{1}}\right)^{m} \prod_{i=1}^{n}\left(x_{i}+1\right) e^{-\theta x_{i}^{2}} \prod_{j=1}^{m}\left(y_{1 j}+1\right) e^{-\theta_{1} y_{1 j}^{2}} .
$$
is

And, the log-likelihood function of the observed samples

$$
\begin{aligned}
\ln L\left(\theta \cdot \theta_{1}\right)= & (m+n) \ln (2)+n \ln (\theta)+m \ln \left(\theta_{1}\right)-n \ln (1+\sqrt{\pi \theta})-m \ln \left(1+\sqrt{\pi \theta_{1}}\right) \\
& -\theta \sum_{i=1}^{n} x_{i}^{2}-\theta_{1} \sum_{j=1}^{m} y_{1 j}^{2}+\sum_{i=1}^{n} \ln \left(x_{i}+1\right)+\sum_{j=1}^{m} \ln \left(y_{1 j}+1\right) .
\end{aligned}
$$

By solving the following equations, the MLE of $\theta$ and $\theta_{2}$ can be obtained:

$$
\begin{gathered}
\frac{\left(\partial \ln L\left(\theta \cdot \theta_{1}\right)\right)}{\partial \theta}=\frac{n}{\theta}-\frac{(n \sqrt{\pi})}{(2 \sqrt{\theta}(1+\sqrt{\pi \theta}))}-\sum_{(i=1)}^{n} x_{i}^{2}=0 \\
\frac{\partial \ln L\left(\theta \cdot \theta_{1}\right)}{\partial \theta_{1}}=\frac{m}{\theta_{1}}-\frac{m \sqrt{\pi}}{2 \sqrt{\theta_{1}}\left(1+\sqrt{\pi \theta_{1}}\right)}-\sum_{j=1}^{m} y_{1 j}^{2}=0 .
\end{gathered}
$$

The MLEs of $\theta$ and $\theta_{1}$ can be obtained, respectively, as

$$
\begin{gathered}
\widehat{\theta}=\frac{1}{6 \pi A^{2}}\left(B+2 A(A+n \pi)+\frac{A^{2}\left(n^{2} \pi^{2}+4 A(A-4 n \pi)\right)}{B}\right), \\
\widehat{\theta}_{1}=\frac{1}{6 \pi C^{2}}\left(D+2 C(C+m \pi)+\frac{C^{2}\left(m^{2} \pi^{2}+4 C(C-4 m \pi)\right)}{D}\right),
\end{gathered}
$$

where $A=\sum_{i=0}^{n} x_{i}^{2} \cdot C=\sum_{j=0}^{m} y_{1 j}^{2}$,

$$
B=\left(8 A^{6}-48 \pi n A^{5}+51 \pi^{2} n^{2} A^{4}-\pi^{3} n^{3} A^{3}+3 \sqrt{3} \pi^{3 / 2} \sqrt{n^{3} A^{7}\left(-16 A^{2}+71 \pi n A-2 \pi^{2} n^{2}\right)}\right)^{1 / 3},
$$

$D=\left(8 C^{6}-48 \pi m C^{5}+51 \pi^{2} m^{2} C^{4}-\pi^{3} m^{3} C^{3}+3 \sqrt{3}\right.$

$\left.\pi^{3 / 2} \sqrt{m^{3} C^{7}\left(-16 C^{2}+71 \pi m C-2 \pi^{2} m^{2}\right)}\right)^{1 / 3}$.

Then, the maximum likelihood estimator of $R$ when the strength $X$ follows $\mathrm{RHN}(\theta)$ distribution and stress $Y$ follows $\operatorname{RHN}\left(\theta_{1}\right)$ distribution is given as

$$
\widehat{R}_{1}=\frac{\left(1+\sqrt{\pi \widehat{\theta}_{1}}+2 \sqrt{\widehat{\theta} \widehat{\theta}_{1}} \operatorname{Tan}^{-1}\left(\sqrt{\left(\hat{\theta} / \widehat{\theta}_{1}\right)}\right)+\left(\sqrt{\pi}\left(\widehat{\theta}-\widehat{\theta}_{1}\right) / \sqrt{\widehat{\theta}+\widehat{\theta}_{1}}\right)-\left(\widehat{\theta}_{1} /\left(\widehat{\theta}+\widehat{\theta}_{1}\right)\right)\right)}{1+\sqrt{\pi \widehat{\theta}}+\sqrt{\pi \widehat{\theta}_{1}}+\pi \sqrt{\widehat{\hat{\theta} \hat{\theta}_{1}}}} .
$$

Similarly, we perform the same steps to find (MLE) in other cases; we can obtain

(i) When the stress $Y_{2}$ that follows the exponential distribution with parameter $\theta_{2}$, the MLE of $R_{2}$ is given as

$$
\widehat{R}_{2}=\frac{\sqrt{\pi}}{2\left(\sqrt{\hat{\theta}}+\sqrt{\pi} \hat{\theta}_{2}\right)}\left(2 \widehat{\theta}-\left(2 \widehat{\theta}-\widehat{\theta}_{2}\right) e^{\left(\widehat{\theta}_{2}^{2} / 4 \hat{\theta}\right)} \operatorname{Erfc}\left(\frac{\widehat{\theta}_{2}}{2 \sqrt{\hat{\theta}}}\right)\right)
$$

where the strength $X$ follows Rayleigh-half-normal distribution with parameter $\theta$. (ii) When the stress $Y_{3}$ that follows Rayleigh distribution with parameter $\theta_{3}$ and the strength $X$ follows Rayleigh-half-normal distribution with parameter $\theta$, the MLE of $R_{3}$ is given as

$$
\widehat{R}_{3}=\frac{\widehat{\theta}}{1+\sqrt{\pi \hat{\theta}}}\left(\frac{1}{\widehat{\theta}}+\frac{\sqrt{\pi}}{\sqrt{\hat{\theta}}}-\frac{2}{2 \widehat{\theta}+\left(1 / \widehat{\theta}_{3}^{2}\right)}-\frac{\sqrt{2 \pi}}{\sqrt{2 \widehat{\theta}+\left(1 / \widehat{\theta}_{3}^{2}\right)}}\right) .
$$

(iii) When the stress $Y_{4}$ that follows half-normal distribution with parameter $\theta_{4}$, the M. L. E of $R_{2}$ is given as 
TABLE 1: Variation in $R_{1}$ when strength and stress has RHN distribution.

\begin{tabular}{lcccccccccc}
\hline$\theta$ & & & & & & $\theta_{1}$ & & & \\
& $\mathbf{0 . 1}$ & $\mathbf{0 . 2}$ & $\mathbf{0 . 3}$ & $\mathbf{0 . 4}$ & $\mathbf{0 . 5}$ & $\mathbf{0 . 6}$ & $\mathbf{0 . 7}$ & $\mathbf{0 . 8}$ & $\mathbf{0 . 9}$ & $\mathbf{1}$ \\
\hline $\mathbf{0 . 1}$ & 0.208 & 0.359 & 0.472 & 0.558 & 0.624 & 0.677 & 0.718 & 0.752 & 0.780 \\
$\mathbf{0 . 2}$ & 0.149 & 0.267 & 0.364 & 0.442 & 0.507 & 0.561 & 0.606 & 0.645 & 0.678 & 0.703 \\
$\mathbf{0 . 3}$ & 0.121 & 0.222 & 0.307 & 0.379 & 0.440 & 0.493 & 0.538 & 0.577 & 0.611 & 0.641 \\
$\mathbf{0 . 4}$ & 0.105 & 0.194 & 0.271 & 0.337 & 0.395 & 0.445 & 0.490 & 0.529 & 0.563 & 0.594 \\
$\mathbf{0 . 5}$ & 0.093 & 0.174 & 0.245 & 0.307 & 0.362 & 0.410 & 0.453 & 0.491 & 0.526 & 0.556 \\
$\mathbf{0 . 6}$ & 0.085 & 0.159 & 0.225 & 0.284 & 0.336 & 0.382 & 0.424 & 0.461 & 0.495 & 0.526 \\
$\mathbf{0 . 7}$ & 0.078 & 0.148 & 0.210 & 0.265 & 0.315 & 0.360 & 0.400 & 0.437 & 0.470 & 0.500 \\
$\mathbf{0 . 8}$ & 0.073 & 0.138 & 0.197 & 0.250 & 0.297 & 0.341 & 0.380 & 0.416 & 0.448 & 0.478 \\
$\mathbf{0 . 9}$ & 0.068 & 0.130 & 0.186 & 0.237 & 0.283 & 0.325 & 0.363 & 0.398 & 0.430 & 0.459 \\
$\mathbf{1}$ & 0.065 & 0.124 & 0.177 & 0.226 & 0.270 & 0.311 & 0.348 & 0.382 & 0.413 & 0.443 \\
\hline
\end{tabular}

$$
\widehat{R}_{4}=\frac{1}{\sqrt{\hat{\theta}}(1+\sqrt{\pi \hat{\theta}})}\left(\frac{2 \hat{\theta} \operatorname{Cot}^{-1}\left(\widehat{\theta}_{2} \sqrt{2 \hat{\theta}}\right)}{\sqrt{\pi}}+\frac{1}{\widehat{\theta}_{4} \sqrt{2+\left(1 / \widehat{\theta} \hat{\theta}_{4}^{2}\right)}}\right),
$$

where the strength $X$ follows Rayleigh-half-normal distribution with parameter $\theta$.

\section{Numerical Evaluation}

In different cases, the system reliability $R$ has evaluated for some specific values of the parameters involved in the expression of $R$.

5.1. Case 1: Strength and Stress Follows RHN Distribution. From Table 1 and Figures 1 and 2, it is noticed that, with the increase in the strength parameter values, the reliability value decreases. If the stress parameter increases, then the value of reliability increases.

5.2. Case 2: Strength Follows RHN Distribution and Stress Follows Exponential Distribution. From Table 2 and Figures 3 and 4, it is observed that if the strength parameter increases then the value of reliability increases. If the stress parameter increases, then the value of reliability increases.

\subsection{Case 3: Strength Follows RHN Distribution and Stress} Follows Rayleigh Distribution. From Table 3 and Figures 5 and 6 , it is noticed that, with increasing the value of the strength and stress parameter, the reliability value decreases.

5.4. Case 4: Strength Follows RHN Distribution and Stress Follows Half-Normal Distribution. From Table 4 and Figures 7 and 8 , it is noticed that, with increasing the value of the strength and stress parameter, the reliability value decreases.

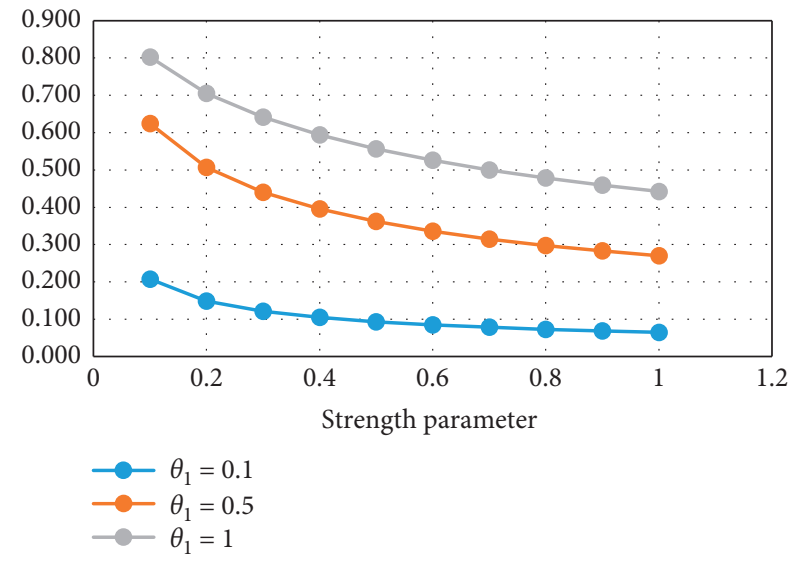

Figure 1: Variation in $R_{1}$ for constant stress.

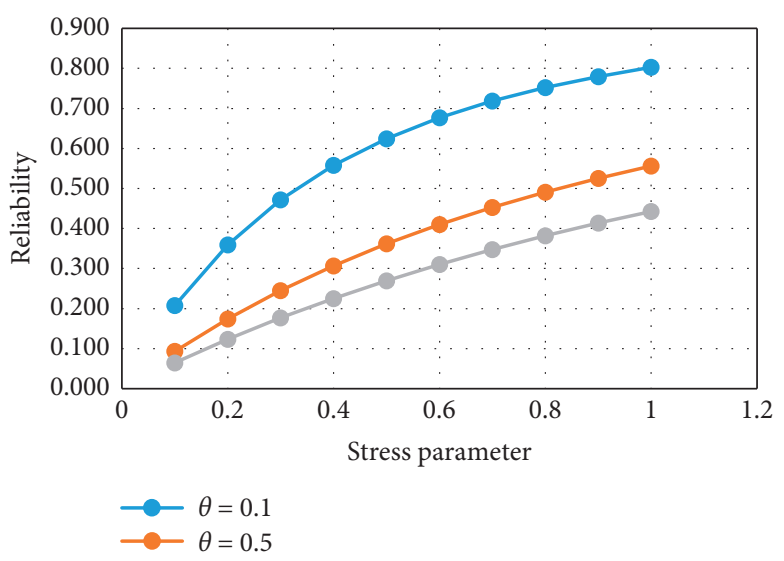

FIgURE 2: Variation in $R_{1}$ for constant strength.

5.5. Simulation Study. In this section, some results are represented depending on Monte-Carlo simulation, for comparing the estimates of $(R)$ performance using MLE and MOM estimators fundamentally for many sample sizes. The following sample sizes are considered; $(n, m)=(5,5),(10$, $10),(20,20),(30,30),(40,40),(50,50)$, and $(100,100)$. From each sample, the estimates are computed for the parameters using MLE and method of moment estimation. Once the 
TABLE 2: Variation in $R_{2}$ when strength has RHN distribution and stress has Exponential distribution.

\begin{tabular}{lcccccccccc}
\hline$\theta$ & & & & & & & & & & \\
& $\mathbf{0 . 1}$ & $\mathbf{0 . 2}$ & $\mathbf{0 . 3}$ & $\mathbf{0 . 4}$ & $\mathbf{0 . 5}$ & $\mathbf{0 . 6}$ & $\mathbf{0 . 7}$ & $\mathbf{0 . 8}$ & $\mathbf{0 . 9}$ & $\mathbf{1}$ \\
\hline $\mathbf{0 . 1}$ & 0.500 & 0.349 & 0.274 & 0.228 & 0.197 & 0.175 & 0.157 & 0.144 & 0.133 \\
$\mathbf{0 . 2}$ & 0.651 & 0.500 & 0.412 & 0.354 & 0.312 & 0.280 & 0.256 & 0.236 & 0.219 & 0.124 \\
$\mathbf{0 . 3}$ & 0.726 & 0.588 & 0.500 & 0.438 & 0.392 & 0.357 & 0.328 & 0.304 & 0.285 & 0.268 \\
$\mathbf{0 . 4}$ & 0.772 & 0.646 & 0.562 & 0.500 & 0.453 & 0.415 & 0.384 & 0.359 & 0.337 & 0.318 \\
$\mathbf{0 . 5}$ & 0.803 & 0.688 & 0.608 & 0.547 & 0.500 & 0.462 & 0.430 & 0.403 & 0.380 & 0.361 \\
$\mathbf{0 . 6}$ & 0.825 & 0.720 & 0.643 & 0.585 & 0.538 & 0.500 & 0.468 & 0.441 & 0.417 \\
$\mathbf{0 . 7}$ & 0.843 & 0.744 & 0.672 & 0.616 & 0.570 & 0.532 & 0.500 & 0.472 & 0.448 & 0.396 \\
$\mathbf{0 . 8}$ & 0.856 & 0.764 & 0.696 & 0.641 & 0.597 & 0.559 & 0.528 & 0.500 & 0.476 \\
$\mathbf{0 . 9}$ & 0.867 & 0.781 & 0.715 & 0.663 & 0.620 & 0.583 & 0.552 & 0.524 & 0.500 & 0.455 \\
$\mathbf{1}$ & 0.876 & 0.795 & 0.732 & 0.682 & 0.639 & 0.604 & 0.573 & 0.545 & 0.521 & 0.500 \\
\hline
\end{tabular}

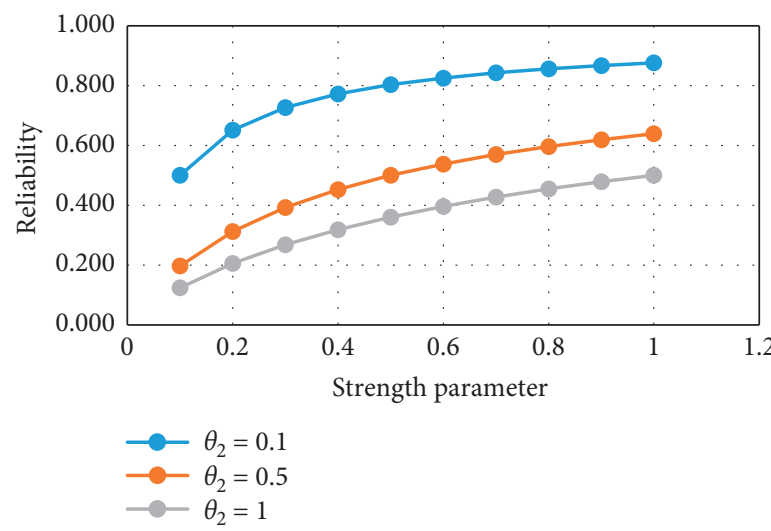

FIgURe 3: Variation in $R_{2}$ for constant stress.

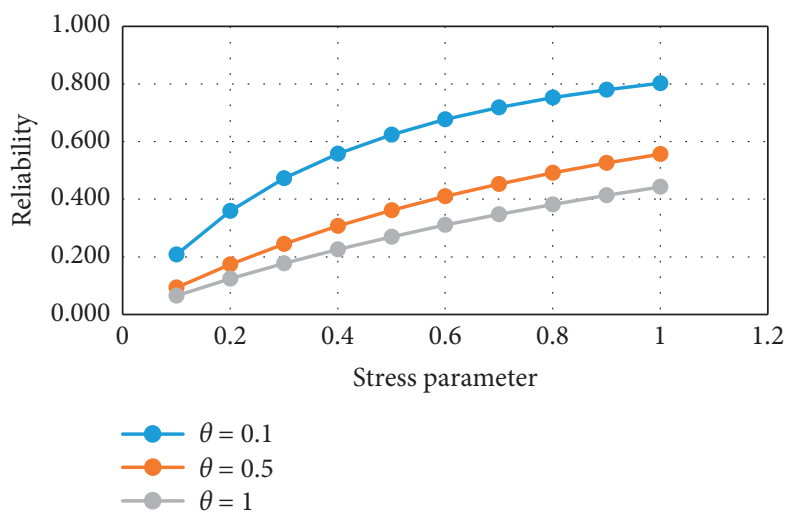

Figure 4: Variation in $R_{2}$ for constant strength.

TABLE 3: Variation in $R_{3}$ when strength has RHN distribution and stress has Rayleigh distribution.

\begin{tabular}{lcccccccccc}
\hline$\theta$ & & & & & & $\theta_{3}$ & & & & \\
& $\mathbf{0 . 1}$ & $\mathbf{0 . 2}$ & $\mathbf{0 . 3}$ & $\mathbf{0 . 4}$ & $\mathbf{0 . 5}$ & $\mathbf{0 . 6}$ & $\mathbf{0 . 7}$ & $\mathbf{0 . 8}$ & $\mathbf{0 . 9}$ & $\mathbf{1}$ \\
\hline $\mathbf{0 . 1}$ & 0.983 & 0.963 & 0.941 & 0.917 & 0.891 & 0.864 & 0.835 & 0.806 & 0.777 \\
$\mathbf{0 . 2}$ & 0.970 & 0.936 & 0.898 & 0.858 & 0.816 & 0.773 & 0.730 & 0.687 & 0.645 & 0.747 \\
$\mathbf{0 . 3}$ & 0.959 & 0.913 & 0.863 & 0.810 & 0.756 & 0.702 & 0.650 & 0.600 & 0.552 & 0.508 \\
$\mathbf{0 . 4}$ & 0.949 & 0.892 & 0.831 & 0.768 & 0.706 & 0.645 & 0.587 & 0.533 & 0.483 & 0.438 \\
$\mathbf{0 . 5}$ & 0.940 & 0.874 & 0.804 & 0.732 & 0.662 & 0.596 & 0.535 & 0.479 & 0.429 & 0.385 \\
$\mathbf{0 . 6}$ & 0.932 & 0.857 & 0.778 & 0.700 & 0.625 & 0.555 & 0.492 & 0.436 & 0.386 & 0.343 \\
$\mathbf{0 . 7}$ & 0.924 & 0.841 & 0.755 & 0.671 & 0.591 & 0.519 & 0.455 & 0.399 & 0.351 & 0.309 \\
$\mathbf{0 . 8}$ & 0.917 & 0.826 & 0.734 & 0.644 & 0.562 & 0.488 & 0.424 & 0.368 & 0.321 & 0.281 \\
$\mathbf{0 . 9}$ & 0.910 & 0.812 & 0.714 & 0.620 & 0.535 & 0.460 & 0.396 & 0.342 & 0.296 & 0.257 \\
$\mathbf{1}$ & 0.903 & 0.799 & 0.695 & 0.598 & 0.511 & 0.435 & 0.372 & 0.318 & 0.274 & 0.238 \\
\hline
\end{tabular}




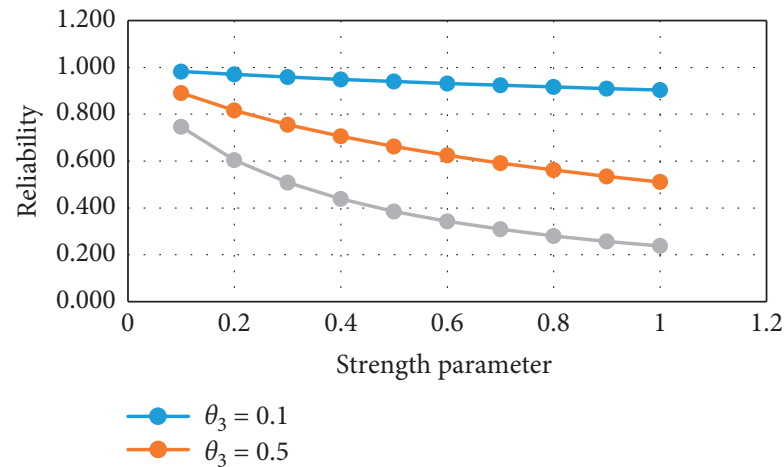

Figure 5: Variation in $R_{3}$ for constant stress.

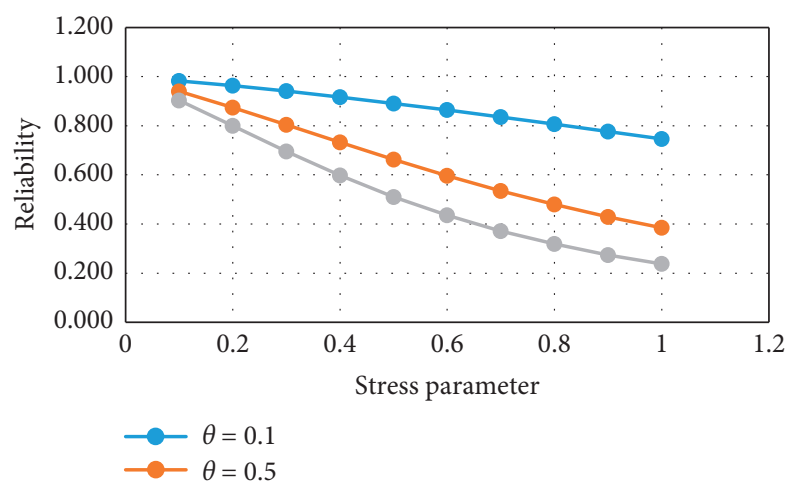

FIgURE 6: Variation in $R_{3}$ for constant strength.

TABLE 4: Variation in $R_{4}$ when strength has RHN distribution and stress has half-normal distribution.

\begin{tabular}{lcccccccccc}
\hline$\theta$ & & & & & & $\theta_{4}$ & & & & \\
& $\mathbf{0 . 1}$ & $\mathbf{0 . 2}$ & $\mathbf{0 . 3}$ & $\mathbf{0 . 4}$ & $\mathbf{0 . 5}$ & $\mathbf{0 . 6}$ & $\mathbf{0 . 7}$ & $\mathbf{0 . 8}$ & $\mathbf{0 . 9}$ & $\mathbf{1}$ \\
\hline $\mathbf{0 . 1}$ & 0.989 & 0.977 & 0.964 & 0.950 & 0.934 & 0.918 & 0.901 & 0.884 & 0.866 & 0.848 \\
$\mathbf{0 . 2}$ & 0.981 & 0.960 & 0.937 & 0.913 & 0.888 & 0.862 & 0.835 & 0.808 & 0.781 & 0.755 \\
$\mathbf{0 . 3}$ & 0.974 & 0.946 & 0.915 & 0.883 & 0.850 & 0.816 & 0.783 & 0.750 & 0.718 & 0.687 \\
$\mathbf{0 . 4}$ & 0.968 & 0.933 & 0.896 & 0.857 & 0.817 & 0.778 & 0.740 & 0.703 & 0.668 & 0.634 \\
$\mathbf{0 . 5}$ & 0.963 & 0.921 & 0.878 & 0.834 & 0.789 & 0.745 & 0.704 & 0.664 & 0.627 & 0.592 \\
$\mathbf{0 . 6}$ & 0.957 & 0.911 & 0.862 & 0.812 & 0.764 & 0.717 & 0.672 & 0.630 & 0.592 & 0.557 \\
$\mathbf{0 . 7}$ & 0.952 & 0.901 & 0.847 & 0.793 & 0.741 & 0.691 & 0.644 & 0.602 & 0.562 & 0.527 \\
$\mathbf{0 . 8}$ & 0.948 & 0.891 & 0.833 & 0.775 & 0.720 & 0.668 & 0.620 & 0.576 & 0.537 & 0.501 \\
$\mathbf{0 . 9}$ & 0.943 & 0.883 & 0.820 & 0.759 & 0.701 & 0.647 & 0.598 & 0.554 & 0.514 & 0.479 \\
$\mathbf{1}$ & 0.939 & 0.874 & 0.808 & 0.744 & 0.683 & 0.628 & 0.578 & 0.533 & 0.494 & 0.459 \\
\hline
\end{tabular}

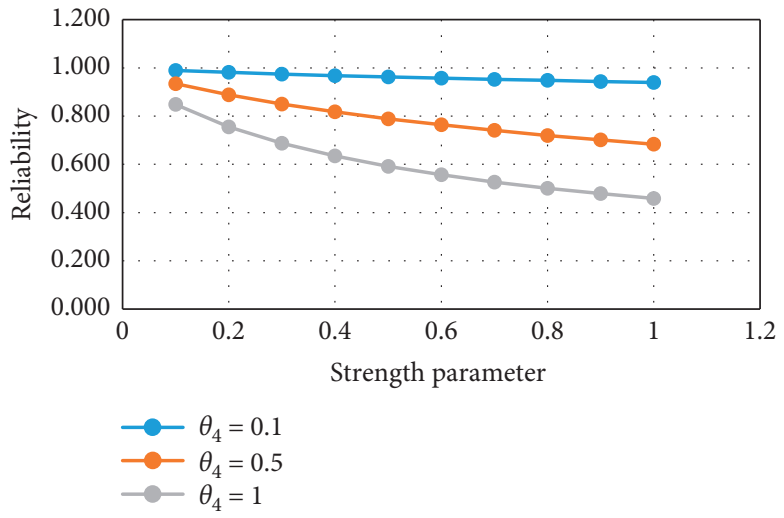

FIGURE 7: Variation in $R_{4}$ for constant stress. 


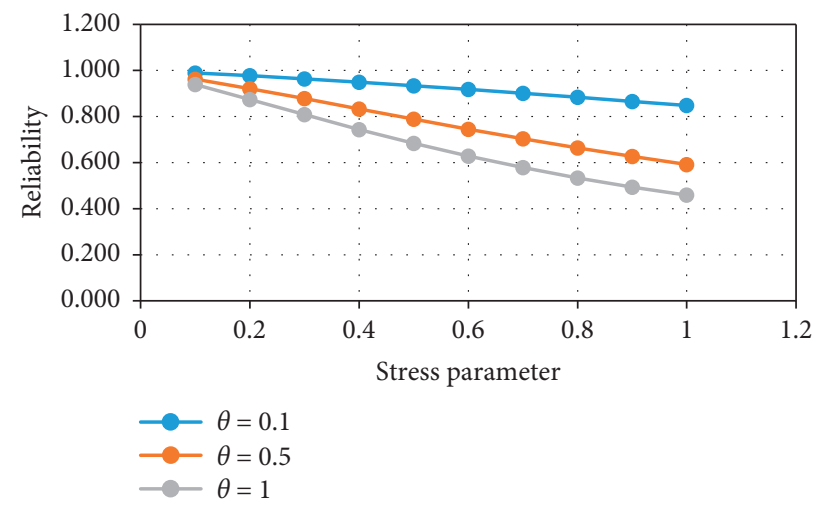

Figure 8: Variation in $R_{4}$ for constant strength.

TABLE 5: Average MSE of the simulated estimates of $R_{1}$.

\begin{tabular}{|c|c|c|c|c|c|c|c|}
\hline \multirow{2}{*}{$\left(\theta \cdot \theta_{1}\right)$} & \multicolumn{7}{|c|}{$(n, m)$} \\
\hline & $(5,5)$ & $(10,10)$ & $(20,20)$ & $(30,30)$ & $(40,40)$ & $(50,50)$ & $(100,100)$ \\
\hline \multirow{2}{*}{$(1,1)$} & 0.0196 & 0.0180 & -0.0181 & -0.0073 & -0.0041 & -0.0034 & 0.0005 \\
\hline & -0.0720 & -0.0318 & -0.0193 & 0.0138 & -0.0084 & 0.0076 & -0.0029 \\
\hline \multirow{2}{*}{$(1,0.5)$} & -0.0594 & -0.0476 & -0.0132 & -0.0120 & -0.0101 & -0.0043 & 0.0048 \\
\hline & -0.0790 & -0.0747 & -0.0643 & -0.0662 & -0.0617 & -0.0658 & -0.0555 \\
\hline \multirow{2}{*}{$(1,1.5)$} & -0.0419 & 0.0262 & 0.0172 & 0.0146 & -0.0063 & 0.0024 & 0.0011 \\
\hline & 0.0678 & 0.0643 & 0.0557 & 0.0478 & 0.0423 & 0.0372 & 0.0370 \\
\hline \multirow{2}{*}{$(1,2)$} & -0.0125 & 0.0115 & 0.0111 & 0.0113 & -0.0073 & 0.0044 & -0.0015 \\
\hline & 0.0800 & -0.0777 & 0.0687 & 0.0663 & 0.0656 & 0.0649 & 0.0638 \\
\hline \multirow{2}{*}{$(0.5,1)$} & 0.0252 & 0.0146 & 0.0122 & -0.0097 & -0.0030 & -0.0018 & 0.0011 \\
\hline & 0.0743 & 0.0737 & 0.0686 & 0.0656 & 0.0654 & 0.0639 & 0.0627 \\
\hline \multirow{2}{*}{$(1.5,1)$} & 0.0594 & -0.0090 & 0.0076 & -0.0064 & 0.0062 & 0.0019 & 0.0019 \\
\hline & -0.0807 & -0.0413 & -0.0402 & -0.0364 & -0.0352 & -0.0164 & 0.0125 \\
\hline \multirow{2}{*}{$(2,1)$} & 0.0153 & 0.0066 & 0.0066 & 0.0061 & 0.0048 & 0.0048 & 0.0045 \\
\hline & -0.0997 & -0.0726 & -0.0719 & -0.0647 & -0.0624 & -0.0618 & -0.0584 \\
\hline
\end{tabular}

TABle 6: Average MSE of the simulated estimates of $R_{1}$.

\begin{tabular}{|c|c|c|c|c|c|c|c|}
\hline \multirow{2}{*}{$\left(\theta \cdot \theta_{1}\right)$} & \multicolumn{7}{|c|}{$(n, m)$} \\
\hline & $(5,5)$ & $(10,10)$ & $(20,20)$ & $(30,30)$ & $(40,40)$ & $(50,50)$ & $(100,100)$ \\
\hline \multirow{2}{*}{$(1,1)$} & 0.0051 & 0.0020 & 0.0020 & 0.0013 & 0.0010 & 0.0005 & 0.0003 \\
\hline & 0.0159 & 0.0104 & 0.0090 & 0.0035 & 0.0024 & 0.0020 & 0.0013 \\
\hline \multirow{2}{*}{$(1,0.5)$} & 0.0123 & 0.0095 & 0.0037 & 0.0033 & 0.0025 & 0.0018 & 0.0009 \\
\hline & 0.0148 & 0.0112 & 0.0051 & 0.0049 & 0.0046 & 0.0044 & 0.0044 \\
\hline \multirow{2}{*}{$(1,1.5)$} & 0.0077 & 0.0072 & 0.0044 & 0.0030 & 0.0022 & 0.0020 & -0.0001 \\
\hline & 0.0135 & 0.0091 & 0.0054 & 0.0051 & 0.0034 & 0.0021 & 0.0020 \\
\hline \multirow{2}{*}{$(1,2)$} & 0.0055 & 0.0050 & 0.0048 & 0.0033 & 0.0022 & 0.0019 & -0.0003 \\
\hline & 0.0115 & 0.0086 & 0.0059 & 0.0059 & 0.0049 & 0.0049 & 0.0009 \\
\hline \multirow{2}{*}{$(0.5,1)$} & 0.0090 & 0.0038 & 0.0038 & 0.0036 & 0.0031 & 0.0024 & 0.0010 \\
\hline & 0.0092 & 0.0087 & 0.0070 & 0.0050 & 0.0050 & 0.0048 & 0.0046 \\
\hline \multirow{2}{*}{$(1.5,1)$} & 0.0147 & 0.0097 & 0.0024 & 0.0024 & -0.0018 & 0.0014 & -0.0008 \\
\hline & 0.0221 & -0.0124 & -0.0090 & 0.0062 & 0.0027 & 0.0022 & 0.0014 \\
\hline \multirow{2}{*}{$(2,1)$} & 0.0121 & 0.0064 & 0.0059 & 0.0030 & 0.0024 & 0.0018 & -0.0003 \\
\hline & 0.0478 & -0.0272 & 0.0256 & -0.0145 & -0.0076 & 0.0050 & 0.0010 \\
\hline
\end{tabular}


parameters are estimated, the estimates of $R_{1}$ is obtained. The average biases of $R_{1}$ is reported in Table 5 and mean squared errors (MSEs) of $R_{1}$ are in Table 6.

The first row includes the average bias of $R_{1}$ using the MLE and second row includes the average bias of $R_{1}$ using the MOM, in each cell.

The first row includes the average MSE of $R_{1}$ using the MLE and second row includes the average MSE of $R_{1}$ using the MOM, in each cell.

\section{Conclusions}

The proposed model in this paper, the stress-strength reliability has been studied for Rayleigh-half normal when the strength $(X)$ follows Rayleigh-half normal distribution, and the stress $(Y)$ takes Rayleigh-half normal distribution, exponential distribution, Rayleigh distribution, and halfnormal distribution. Based on the computations and graphs, (i) it has been noticed that when the stress parameter is increased, the reliability value lowers, and when the strength parameter is increased, the reliability value increases. The numerical assessment demonstrates that increasing the stress parameter decreases the dependability value in case (ii), whereas increasing the strength parameter increases the reliability value. In cases (iii) and (vi), increasing the stress parameter decreases the reliability value, whereas increasing the strength parameter increases it. A comparison is carried out between two methods of reliability estimation $R=P(X>Y)$ when $(Y)$ and $(X)$ both follow Rayleigh-half normal distributions for various parameters scale. We provide MLE and MOM procedure for estimating the unknown parameters that are used for reliability estimation $(R)$. Based on the simulation findings, we can conclude that MLE outperforms MOM in terms of average bias and average MSE for a variety of parameter choices.

\section{Data Availability}

All data used to support the findings of the study are available within the article.

\section{Conflicts of Interest}

The authors declare that they have no conflicts of interest.

\section{References}

[1] J. D. Church and B. Harris, "The estimation of reliability from stress-strength relationships," Technometrics, vol. 12, no. 1, pp. 49-54, 1970.

[2] J. G. Surles and W. J. Padgett, "Inference for reliability and stress-strength for scald Burr X distribution," Lifetime Data Analysis, vol. 7, no. 2, pp. 187-200, 2001.

[3] M. Z. Raqab and D. Kundu, "Comparison of different estimates of $P(Y<X)$ for a scaled Burr type $X$ distribution," Communications in Statistics - Simulation and Computation, vol. 34, no. 2, pp. 465-483, 2005.

[4] N. Mokhlis, "Reliability of a stress-strength model with Burr type III distributions," Communications in Statistics-Theory and Methods, vol. 34, no. 7, pp. 1643-1657, 2005.
[5] B. Saraçoğlu, İ. Kınacı, and D. Kundu, "On estimation of $(P(Y$ $<X)$ for exponential distribution under progressive type-II censoring," Journal of Statistical Computation and Simulation, vol. 82, no. 5, pp. 729-744, 2011.

[6] S. Kotz, Y. Lumelskii, and M. Pensky, The Stress-Strength Model and its Generalizations: Theory and Applications, World Scientific Publishing, Singapore, 2003.

[7] H. Khan Adil and T. R. Jan, "On estimation of reliability function of Consul and Geeta distributions," International Journal of Advanced Scientific and Technical Research, vol. 4, no. 4, pp. 96-105, 2014.

[8] O. Akman, P. Sansgiry, and M. C. Minnotte, "On the estimation of reliability based on mixture inverse Gaussian distributions," Applied Statistical Science, Vol. IV, Nova Science Publishers, New York, NY, USA, 1999.

[9] E. K. AI-Hussaini, "Bayesian prediction under a mixture of two exponential components model based on type 1 censoring," Journal of Applied Statistical Science, vol. 8, pp. 173-185, 1999.

[10] M. M. M. El-Din, M. M. Amein, H. E. El-Attar, and E. H. Hafez, "Symmetric and asymmetric bayesian estimation for lindley distribution based on progressive first failure censored data," Mathematical Sciences Letters, vol. 6, no. 3, pp. 255-260, 2017, p.

[11] J. Zhao, Z. Ahmad, E. Mahmoudi, E. H. Hafez, and M. M. Mohie El-Din, "A new class of heavy-tailed distributions: modeling and simulating actuarial measures," Complexity, vol. 2021, Article ID 5580228, , 2021.

[12] H. M. Almongy, E. Almetwally, H. M. Aljohani, A. S. Alghamdi, and E. H. Hafez, "A new extended Rayleigh distribution with applications of COVID-19 data," Results in Physics, vol. 23, no. 60, p, Article ID 104012, 2021.

[13] H. M. Almongy, F. Y. Alshenawy, E. M. Almetwally, and D. A. Abdo, "Applying transformer insulation using Weibull extended distribution based on progressive censoring scheme," Axioms, vol. 10, no. 2, p. 100, 2021.

[14] M. H Abu-Moussa, A. M. Abd-Elfattah, and E. H Hafez, "Estimation of stress-strength parameter for Rayleigh distribution based on progressive type-II censoring," Information Sciences Letters, vol. 10, no. 1, p. 12, 2021.

[15] M. M. E. Abd El-Monsef and M. M. Abd El-Raouf, "Rayleighhalf normal distribution for modeling tooth movement data," Journal of Biopharmaceutical Statistics, vol. 30, no. 3, pp. 481-494, 2020.

[16] S. Nadarajah, "The exponentiated Gumbel distribution with climate application," Environmetrics, vol. 17, no. 1, pp. 13-23, 2006.

[17] W. Barreto-Souza, A. L. De Morais, and G. M. Cordeiro, "The Weibull-geometric distribution," Journal of Statistical Computation and Simulation, vol. 81, no. 5, pp. 645-657, 2011.

[18] J. Milan and R. Vesna, "Estimation of $p\{x<y\}$ for gamma exponential model," Yugoslav Journal of Operations Research, vol. 24, no. 2, pp. 283-291, 2014.

[19] L. Greco and L. Ventura, "Robust inference for the stressstrength reliability," Statistical Papers, vol. 52, no. 4, pp. 773-788, 2011. 